\title{
A SINGULAR INTEGRAL EQUATION METHOD FOR EXAMINING ASYMPTOTIC SOLUTIONS OF A KINKED CRACK WITH INFINITESIMAL KINK LENGTH
}

\author{
Y. Z. CHEN, X. Y. LIN AND Z. X. WANG
}

\begin{abstract}
This paper investigates the singular integral equation method for examining the stress intensity factor and the T-stress in the asymptotic solution of a kinked crack with an infinitesimal kink length. A numerical technique for the branch crack problem is introduced, which depends upon distribution of dislocation along the crack face. The technique reduces the branch crack problem to the solution of a singular integral equation. The kinked cracked problem can be considered as a particular case of the branch crack, and this problem can be solved by using the suggested technique. It is found from the computed results that the available asymptotic solution can give qualitatively correct results for stress intensity factors and the T-stress. In addition, the available asymptotic solution can only give sufficiently accurate results in a narrow range of the length of the kinked portion and the inclined kink angle.
\end{abstract}

\section{Introduction}

Williams [1957] investigated the stress distribution near a crack tip. In the notation of [Rice 1974], the nonsingular term in the Williams expansion is denoted as the T-stress and can be regarded as the stress acting parallel to the crack flanks. The T-stress evaluation may have engineering application in the following fields: determining the plastic zone near the crack tip in the case of small scale yielding [Larsson and Carlsson 1973; Betegón and Hancock 1991] and determining directional stability for the crack growth path [Rice 1974; Melin 2002].

The T-stress evaluation has attracted much attention from many investigators. Using the dislocation distribution method, Broberg [2005] solved several T-stress problems. Stress intensity factors and T-stress solutions for components containing cracks were computed by application of the boundary collocation method (BCM) with the fracture mechanics weight function for the stress intensity factor (SIF) and a Green's function for the T-stress [Fett 2001]. The obtained solutions were limited to a line crack in a circular plate.

Using the hybrid crack element (HCE), Karihaloo and Xiao [2001] evaluated the higher order terms in the stress distribution of a three-point bend beam. The coefficients of the first five terms of the crack tip asymptotic field are computed using a HCE. Those coefficients include the T-stress component. Xiao and Karihaloo [2002] studied the problem for an edge crack in a finite plate with a wedge force on the crack face. The problem was reduced to the problem of a traction free edge crack with loading on the outer boundary. The usage of the Williams expansion and the BCM gave the final solution. Yang and Ravi-Chandar [1999] developed a stress difference method to evaluate the T-stress in the crack problem.

Keywords: kinked crack, stress intensity factors, T-stress, asymptotic solution, singular integral equation. 
It was proved that the limit of the difference of two normal stress components ahead of the crack tip would give the T-stress.

Evaluation of the SIFs and the T-stress in the slightly kinked crack was subject to much attention from many investigators [Lo 1978; Cotterell and Rice 1980; Melin 1986; Fett et al. 2006; Li and Xu 2007]. The Melin transform is used for the solution of a kinked crack [Melin 1994]. A solution was presented for the SIFs at the tip of a slightly curved or kinked crack. The solution is accurate to first order in the deviation of the crack face from a straight line [Cotterell and Rice 1980]. The influence of the T-stress on the stability of crack growth was investigated. The straight path is shown to be stable under mode I loading for $T<0$ [Cotterell and Rice 1980].

Fett et al. [2006] studied the T-stress for a kinked crack and suggested a Green's function to solve the problem. The formulation was based on an edge crack in a rectangular plate. By using asymptotic analysis and the Westergaard stress function method, $\mathrm{Li}$ and $\mathrm{Xu}$ [2007] proposed approximate analytical formulas for the T-stress and the SIFs for a crack with an infinitesimal kink.

The mentioned studies depend on some assumptions. For example, normally we must investigate the SIFs and the T-stress at the kinked crack tip of the kinked crack. However, in asymptotic analysis the researcher generally obtains the SIFs and the T-stress from a semiinfinite crack with loading on some interval on the crack. All mentioned assumptions couldn't be proved theoretically. However, many researchers recognized that those formulations are true when the kinked crack length $d \rightarrow 0$ and the kinked angle $\theta$ keeps up a rather small value, for example $\theta \leq 15^{\circ}$.

In reality, only small crack kink angles were observed for isotropic materials. Large crack kink angles are almost impossible because of the crack branch possibility. Some researchers believe that the maximum crack branch angle is around 60 degrees so it is meaningful to study crack kink angles less than 30 degrees. In this paper, the assumed kink angle is up to 60 degrees. Clearly, the obtained computed results cover the case of a small kink angle.

Two singular point finite elements were developed for the analysis of kinked cracks [Dutta et al. 1991]. The suggested method could reflect the weaker stress singularity at the corner portion of a kinked crack.

In this paper, a numerical examination for the asymptotic solution of a kinked crack with an infinitesimal kink length is carried out. It is assumed that the main crack has a length $b$, and the kinked portion has a length $d$ with an inclined angle $\theta$. If $d / b$ takes a very small value, for example if $d / b=0.001$, and the usual singular integral equation method is used, the kinked portion will not share even one integration point after discretization of the integral equation. Since all the collocation points are placed on the main crack portion, the nature of the kinked portion cannot be reflected. Therefore, the technique suggested by Chen [2004] is no longer useful in the case of an infinitesimal kink length.

In this study, the kinked crack problem is considered as a particular case of the branch crack problem. The branch crack problem can be modeled by a continuous distribution of dislocations along the branches. In addition, a singular integral equation is obtained from this modeling. The number for integration divisions can be assumed for an individual branch even if the branch length is extremely short. Therefore, the kinked problem with an infinitesimal kinked length can be solved numerically. Finally, for the three loading case, $\sigma_{x}^{\infty}=p, \sigma_{y}^{\infty}=p$, or $\sigma_{x y}^{\infty}=-p$, the computed results are compared with those obtained from the asymptotic solution. Therefore, the asymptotic solution for the kinked problems with an infinitesimal kinked length is fully examined. 


\section{Asymptotic solution for a kinked crack with an infinitesimal kink length}

Some basic equations for the singular stress field at the crack tip are introduced below. The stress distribution near a crack tip was investigated early by Williams [1957]. In polar coordinates $(r, \theta)$, the stress components $\sigma_{i j}$ can be expressed by (see Figure 1a)

$$
\left[\begin{array}{cc}
\sigma_{x} & \sigma_{x y} \\
\sigma_{x y} & \sigma_{y}
\end{array}\right]=\frac{K_{1}}{\sqrt{2 \pi r}}\left[\begin{array}{ll}
f_{11}(\theta) & f_{12}(\theta) \\
f_{12}(\theta) & f_{22}(\theta)
\end{array}\right]+\frac{K_{2}}{\sqrt{2 \pi r}}\left[\begin{array}{ll}
g_{11}(\theta) & g_{12}(\theta) \\
g_{12}(\theta) & g_{22}(\theta)
\end{array}\right]+\left[\begin{array}{ll}
T & 0 \\
0 & 0
\end{array}\right]
$$

where the first two terms in the expansion form are singular at the crack tip, $K_{1}$ and $K_{2}$ denote the mode I and II SIFs respectively, and the functions $f_{i j}(\theta)$ and $g_{i j}(\theta)$ represent the angular distributions of stresses near the crack tip. In addition, the third term is finite and bounded. In the notation of [Rice 1974], the third term is denoted as the T-stress.

In Equation (1), the term $O\left(r^{1 / 2}\right)$ has been neglected for clarity. In addition, the angular distribution can be expressed as [Williams 1957]

$$
\left\{\begin{array}{l}
f_{11} \\
f_{12} \\
f_{22}
\end{array}\right\}=\cos \left(\frac{\theta}{2}\right)\left\{\begin{array}{c}
1-\sin \left(\frac{\theta}{2}\right) \sin \left(3 \frac{\theta}{2}\right) \\
\sin \left(\frac{\theta}{2}\right) \cos \left(3 \frac{\theta}{2}\right) \\
1+\sin \left(\frac{\theta}{2}\right) \sin \left(3 \frac{\theta}{2}\right)
\end{array}\right\}, \quad\left\{\begin{array}{l}
g_{11} \\
g_{12} \\
g_{22}
\end{array}\right\}=\left\{\begin{array}{c}
-\sin \left(\frac{\theta}{2}\right)\left[2+\cos \left(\frac{\theta}{2}\right) \cos \left(3 \frac{\theta}{2}\right)\right] \\
\cos \left(\frac{\theta}{2}\right)\left[1-\sin \left(\frac{\theta}{2}\right) \sin \left(3 \frac{\theta}{2}\right)\right] \\
\sin \left(\frac{\theta}{2}\right) \cos \left(\frac{\theta}{2}\right) \cos \left(3 \frac{\theta}{2}\right)
\end{array}\right\} .
$$

An asymptotic solution for a kinked crack with an infinitesimal kink length was suggested previously. The SIFs and the T-stress evaluation after crack kinking in two-dimensional elastic solids were studied [Cotterell and Rice 1980; Fett et al. 2006; Li and Xu 2007]. Contributions from the T-stress before crack kinking to the T-stress and SIFs of the kinked crack are clearly described. It is assumed that the main crack under remote loading, the SIFs, and the T-stress at the crack tip B are denoted by $K_{1}^{m}, K_{2}^{m}$, and $T^{m}$, respectively (see Figure 1b). After kinking, the SIFs and the T-stress at the kinked tip C are denoted by $K_{1}^{k}, K_{2}^{k}$, and $T^{k}$ (see Figure 1c), respectively. After some manipulation, Li and Xu [2007] obtained the following results:

$$
\begin{aligned}
K_{1}^{k} & =c_{11}(\theta) K_{1}^{m}+c_{12}(\theta) K_{2}^{m}+2 T^{m} \sqrt{\frac{2 d}{\pi}} \sin ^{2} \theta . \\
K_{2}^{k} & =c_{21}(\theta) K_{1}^{m}+c_{22}(\theta) K_{2}^{m}-2 T^{m} \sqrt{\frac{2 d}{\pi}} \sin \theta \cos \theta . \\
T^{k} & =\sqrt{\frac{1}{2 \pi d}}\left(N_{1}(\theta) K_{1}^{m}+N_{2}(\theta) K_{2}^{m}\right)+n(\theta) T^{m},
\end{aligned}
$$

where $d$ denotes the length of kinked crack, $\theta$ the kinked angle (see Figure 1c), and

$$
\begin{aligned}
& c_{11}(\theta)=\frac{(1+\cos \theta) \cos (\theta / 2)}{2}, \quad c_{22}(\theta)=\frac{(3 \cos \theta-1) \cos (\theta / 2)}{2}, \\
& c_{12}(\theta)=-\frac{3(1+\cos \theta) \sin (\theta / 2)}{2}, \quad c_{21}(\theta)=\frac{(1+\cos \theta) \sin (\theta / 2)}{2}, \\
& N_{1}(\theta)=\sin \theta \sin \left(\frac{\theta}{2}\right), \quad N_{2}(\theta)=(1+3 \cos \theta) \sin \left(\frac{\theta}{2}\right), \quad n(\theta)=\cos 2 \theta .
\end{aligned}
$$




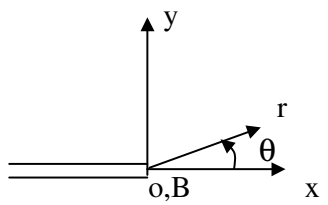

(a)

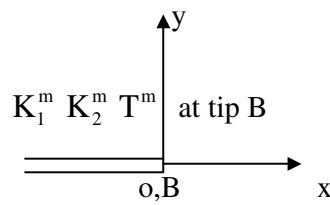

(b)

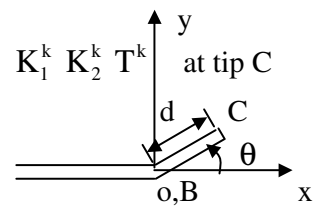

(c)

Figure 1. (a) A semiinfinite crack; (b) a main crack with loading $K_{1}^{m}, K_{2}^{m}$, and $T^{m}$; and (c) a kinked crack emanating from the main crack.

Note that the four functions $c_{11}(\theta), c_{12}(\theta), c_{21}(\theta)$, and $c_{22}(\theta)$ have also been suggested previously [Cotterell and Rice 1980]. It is expected that the suggested formulas are valid for the case of $d \rightarrow 0$, or if the length of the kinked portion is sufficient small.

Clearly, the asymptotic solution can only give an approximate solution for the kinked crack problem. This can be seen from the following analysis. It is assumed that the kinked crack with a small kink length $d$ and an inclined angle $\theta$ is surrounded by a stress field defined by a mode I SIF, or $K_{1}^{m} \neq 0, K_{2}^{m}=0$, and $T^{m}=0$ (see Figure 2a). The original problem shown by Figure $2 \mathrm{a}$ can be considered as a superposition of the two problems shown by Figures $2 b$ and $2 c$. In the problem shown by Figure $2 b$, the main crack has a loading $K_{1}^{m} \neq 0, K_{2}^{m}=0$, and $T^{m}=0$. However, in the problem shown by Figure $2 \mathrm{c}$, the kinked crack has a loading on the kinked portion, or along the interval BC.

Clearly, from Equations (1) and (2), the stress singular distributions along the line BC in $(r, \theta)$ coordinates can be easily evaluated (see Figure 2b):

$$
\sigma_{r}=\frac{K_{1}^{m}}{\sqrt{2 \pi r}} c_{11}(\theta), \quad \sigma_{r} \theta=\frac{K_{1}^{m}}{\sqrt{2 \pi r}} c_{21}(\theta)
$$

where $c_{11}(\theta)$ and $c_{21}(\theta)$ are defined in (6) and (7).

After making the substitution $r=d-s$, the loading on the kinked portion in Figure $2 \mathrm{c}$ will be

$$
\sigma_{r^{*}}=-\sigma_{r}=-\frac{K_{1}^{m}}{\sqrt{2 \pi(d-s)}} c_{11}(\theta), \quad \sigma_{r \theta^{*}}=-\sigma_{r \theta}=-\frac{K_{1}^{m}}{\sqrt{2 \pi(d-s)}} c_{21}(\theta) .
$$

An approximation was introduced as follows [Cotterell and Rice 1980; Li and Xu 2007]. The kinked crack shown by Figure $2 \mathrm{c}$ was approximated by a semiinfinite crack shown by Figure $2 \mathrm{~d}$ with the loading applied on the interval $(0<s<d)$. Therefore, the SIFs at the kinked tip $C$ can be evaluated by

$$
K_{1}^{k}=-\sqrt{\frac{2}{\pi}} \int_{0}^{d} \frac{\sigma_{r^{*}} d s}{\sqrt{s}}=K_{1}^{m} c_{11}(\theta), \quad K_{2}^{k}=-\sqrt{\frac{2}{\pi}} \int_{0}^{d} \frac{\sigma_{r \theta^{*}} d s}{\sqrt{s}}=K_{1}^{m} c_{21}(\theta) .
$$

This result coincides with that obtained from Equations (3), (4), (6), and (7) in the case of $K_{1}^{m} \neq 0$, $K_{2}^{m}=0$, and $T^{m}=0$ [Cotterell and Rice 1980; Li and Xu 2007].

A similar approximation was suggested to consider the influence functions

$$
2 \sqrt{\frac{2 d}{\pi}} \sin ^{2} \theta, \quad-2 \sqrt{\frac{2 d}{\pi}} \sin \theta \cos \theta,
$$

and $n(\theta)$ in (3)-(5), which represent the influence for $K_{1}^{k}, K_{2}^{k}$, and $T^{k}$ caused by $T^{m}$. 


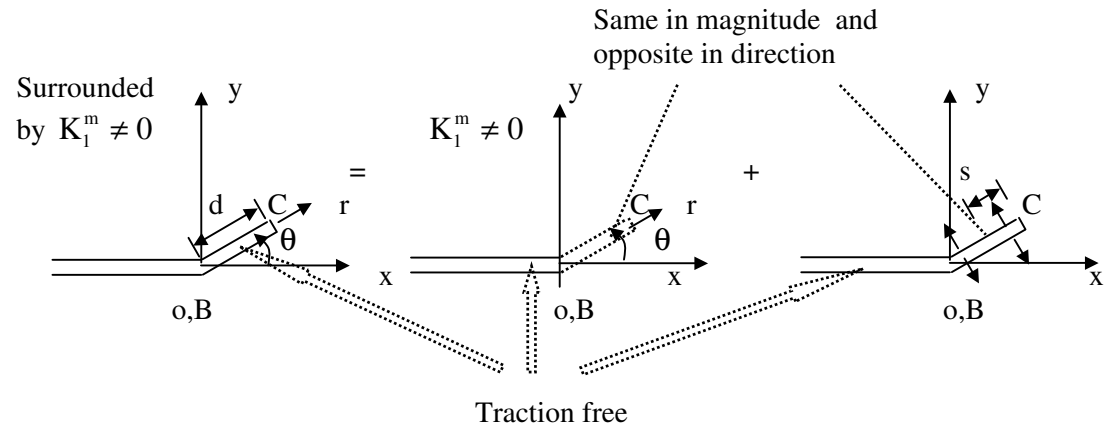

(a)

(b)

(c)

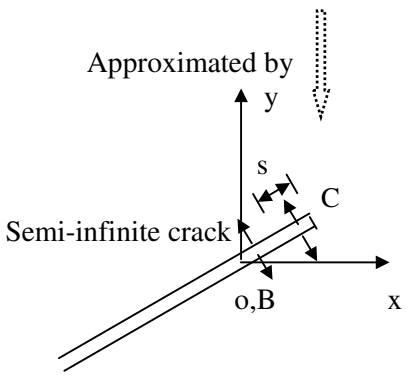

(d)

Figure 2. Superposition method in the kinked problem: (a) a kinked crack surrounded by the stress field defined by loading $K_{1}^{m}$, (b) a main crack with loading $K_{1}^{m}$, (c) a kinked crack with loading on the kinked portion $\mathrm{BC}$, and (d) a semiinfinite crack with loading on the interval BC.

As mentioned above, the boundary value problem shown by Figure $2 \mathrm{c}$ was approximated by the problem shown by Figure $2 \mathrm{~d}$. Clearly, the most difficult point is to evaluate the deviation caused by this approximation. In addition, this difficult problem cannot be solved theoretically. A particular advantage of the asymptotic solution for kinked crack problem is it provides some equations for the SIFs and the T-stress in an explicit form. However, since many assumptions were used in the derivation, the accuracy of the asymptotic solution is generally not easy to judge. Naturally, it is necessary to propose a numerical examination for the asymptotic solution, particularly, for the case of an infinitesimal kink length.

\section{Formulation for the solution of the branch crack problem}

In the following, a numerical method for solving the branch crack problem is suggested. The problem is shown in Figure 3a, for example, a crack problem with three branches. It is assumed that the applied tractions are identical in magnitude and opposite in direction on the both sides of the crack face. The problem can be modeled by a dislocation distribution along the prospective site of the branches (see Figure $3 b$ ). In this case, even though some branches have smaller lengths, we can assume a suitable number of integration divisions for those branches. For example, in Figure 3, for two shorter branches, the branches $\mathrm{BD}$ and $\mathrm{BC}$, we can assume $M_{1}=3$ and $M_{2}=5$ (the numbers of integration divisions), and we can take $M_{3}=15$ for longer branch. The kinked crack (with two branches) is a particular case of the branch crack problem. Therefore, the numerical solution based on the branch crack problem can be used for the kinked crack problem.

For evaluating the SIFs and the T-stress in the branch crack problem, a detailed formulation is introduced below. The complex variable function method plays an important role in plane elasticity. The fundamentals of this method are introduced. In the method, the stresses $\left(\sigma_{x}, \sigma_{y}, \sigma_{x y}\right)$, the resultant forces $(X, Y)$, and the displacements $(u, v)$ are expressed in terms of complex potentials $\phi(z)$ and $\psi(z)$ such that [Muskhelishvili 1953]

$$
\sigma_{x}+\sigma_{y}=4 \operatorname{Re} \phi^{\prime}(z)
$$




$$
\begin{aligned}
\sigma_{y}-\sigma_{x}+2 i \sigma_{x y} & =2\left[\bar{z} \phi^{\prime \prime}(z)+\psi^{\prime}(z)\right], \\
f=-Y+i X & =\phi(z)+z \overline{\phi^{\prime}(z)}+\overline{\psi(z)}, \quad 2 G(u+i v)=\kappa \phi(z)-z \overline{\phi^{\prime}(z)}-\overline{\psi(z)},
\end{aligned}
$$

where a bar over a function denotes the conjugate of the function, $G$ is the shear modulus of elasticity, $\kappa=(3-v) /(1+v)$ in the plane stress problem, $\kappa=3-4 v$ in the plane strain problem, $v$ is the Poisson's ratio, and $i=\sqrt{-1}$ denotes the unit imaginary value.

Except for the physical quantities mentioned above, from Equation (13) two derivatives in specified direction are introduced as follows: [Savruk 1981]

$$
\begin{aligned}
& J_{1}(z)=\frac{d}{d z}\{-Y+i X\}=\Phi(z)+\overline{\Phi(z)}+\frac{d \bar{z}}{d z}\left(z \overline{\Phi^{\prime}(z)}+\overline{\Psi(z)}\right)=\sigma_{N}+i \sigma_{N T}, \\
& J_{2}(z)=2 G \frac{d}{d z}\{u+i v\}=\kappa \Phi(z)-\overline{\Phi(z)}-\frac{d \bar{z}}{d z}\left(z \overline{\Phi^{\prime}(z)}+\overline{\Psi(z)}\right)=(\kappa+1) \Phi(z)-J_{1} .
\end{aligned}
$$

It is easy to verify that $J_{1}=\sigma_{N}+i \sigma_{N T}$ denotes the normal and shear tractions along the segment $\overline{z, z+d z}$. Secondly, the $J_{1}$ and $J_{2}$ values depend not only on the position of a point $z$, but also on the direction of the segment $d \bar{z} / d z$ (see Figure 4o).

For evaluating the T-stress at the branch tips with remote loading, it is suitable to use the superposition method. The original problem is shown in Figure 4o. The remote tractions are denoted as $\sigma_{x}^{\infty}, \sigma_{y}^{\infty}$, and $\sigma_{x y}^{\infty}$. The original field can be considered as a superposition of a uniform field and a perturbation field, which are shown by Figures $4 \mathrm{a}$ and $4 \mathrm{~b}$, respectively. Here and after, the subscript $(u)$ is used for the uniform field, and the subscript ( $p$ ) for the perturbation field (see Figures $4 a$ and $4 b$ ).

Clearly, the T-stress at the $j$-th branch tip $A_{j}$ can be expressed as

$$
T_{j}=T_{j(u)}+T_{j(p)},
$$

where $T_{j(u)}$ and $T_{j(p)}$ denote the T-stress at tip $A_{j}$ from the uniform field and the perturbation field, respectively. It is seen that

$$
T_{j(u)}=\sigma_{T(u)}\left(t_{A_{j}}\right),
$$

where $\sigma_{T(u)}\left(t_{A_{j}}\right)$ denote the $\sigma_{T}$ component at the branch tip $A_{j}$ in the uniform field (see Figure 4a).

Clearly, since the tractions on the crack face in the perturbation field are opposite to those tractions on the perspective site of crack in the uniform field, the boundary tractions applied on the $j$-th branch in

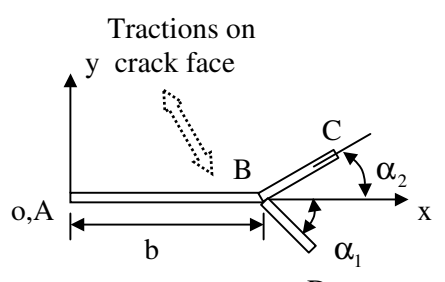

(a)

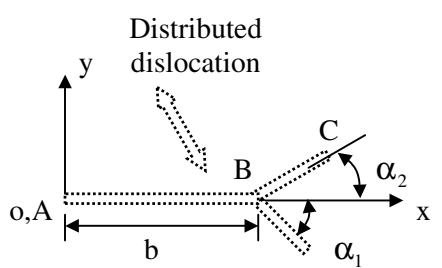

(b)

Figure 3. (a) A branch crack with loading on the crack face, and (b) a branch crack modeled by distributed dislocation. 
(o)

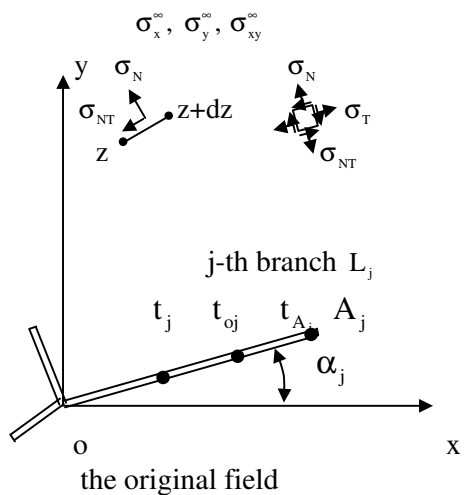

$\sigma_{x}^{\infty}, \sigma_{y}^{\infty}, \sigma_{x y}^{\infty}$

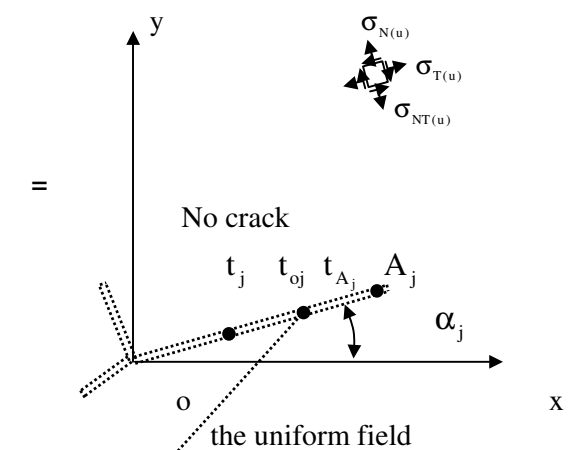

(a)

Same in magnitude and
opposite in direction for $\sigma_{\mathrm{N}}, \sigma_{\mathrm{NT}}$

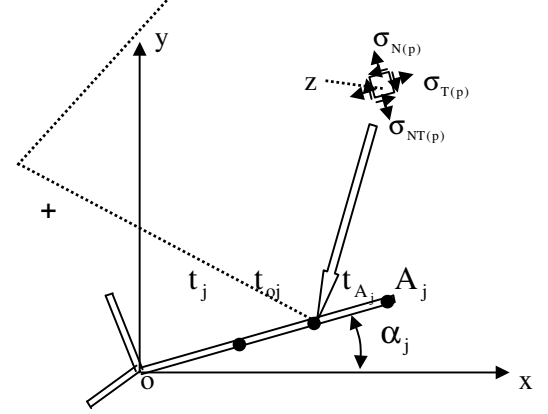

the perturbation field

(b)

Figure 4. Superposition method for the branch crack problem: $(o)$ a branch crack in an infinite plate, with $o$ the original field; $(a)$ a perfect plate with remote loading, with subscript $(u)$ denoting the uniform stress field; and $(b)$ a branch crack with loading on the crack face, with subscript $(p)$ denoting the perturbation stress field.

the perturbation field will be

$$
\sigma_{N(p)}\left(t_{o j}\right)+i \sigma_{N T(p)}\left(t_{o j}\right)=-\left(\sigma_{N(u)}\left(t_{o j}\right)+i \sigma_{N T(u)}\left(t_{o j}\right)\right) \quad\left(t_{o j} \in L_{j} ; j=1,2, \ldots, N\right) .
$$

In order to evaluate the T-stress in the branch crack problem, it is necessary to derive the relevant integral equation beforehand. In the perturbation field, the remote tractions are zero, and the applied tractions on branches are as follows (see Figure $4 b)$ :

$$
\begin{aligned}
\sigma_{N}\left(t_{o j}\right)+i \sigma_{N T}\left(t_{o j}\right) & =\sigma_{N(p)}\left(t_{o j}\right)+i \sigma_{N T(p)}\left(t_{o j}\right) \\
& =-\left(\sigma_{N(u)}\left(t_{o j}\right)+i \sigma_{N T(u)}\left(t_{o j}\right)\right) \quad\left(t_{o j} \in L_{j} ; j=1,2, \ldots, N\right),
\end{aligned}
$$

where $\sigma_{N}\left(t_{o j}\right)$ and $\sigma_{N T}\left(t_{o j}\right)$ denote the normal and shear tractions along the $j$-th branch or $L_{j}$, and $\sigma_{N(p)}\left(t_{o j}\right)$ and $\sigma_{N T(p)}\left(t_{o j}\right)$ denote the given normal and shear tractions along the $j$-th branch (see Figure 4b).

It was proved that the complex potentials for this field could be expressed as [Chen and Hasebe 1995]

$$
\phi^{\prime}(z)=\frac{H}{2 \pi z}+\frac{1}{2 \pi} \sum_{k=1}^{N} \int_{L_{k}} \frac{g_{k}^{\prime}(t) d t}{t-z}, \quad \phi^{\prime \prime}(z)=-\frac{H}{2 \pi z^{2}}+\frac{1}{2 \pi} \sum_{k=1}^{N} \int_{L_{k}} \frac{g_{k}^{\prime}(t) d t}{(t-z)^{2}},
$$




$$
\psi^{\prime}(z)=\frac{\bar{H}}{2 \pi z}+\frac{1}{2 \pi} \sum_{k=1}^{N} \int_{L_{k}} \frac{\overline{g_{k}^{\prime}(t)} d \bar{t}}{t-z}-\frac{1}{2 \pi} \sum_{k=1}^{N} \int_{L_{k}} \frac{\bar{t} g_{k}^{\prime}(t) d t}{(t-z)^{2}},
$$

where $g_{k}^{\prime}(t)$ denotes the dislocation distribution along the $k$-th branch. Here, $H\left(H=H_{1}+i H_{2}\right)$ is a concentrated dislocation placed at the origin. The reason for assuming the complex potentials shown by Equations (20) and (21) will be expressed later.

Physically, the function $g_{k}^{\prime}(t)$ represents the dislocation distribution along the $k$-th crack. Thus, integration to this function will give the crack opening displacement function, or the displacement discontinuity across the crack.

By substituting (20) and (21) into (14), letting the point $z$ approach a point $t_{o_{j}} \in L_{j}$ on the $j$-th branch (see Figure 4b), and using the Plemelj formula for the Cauchy-type integral [Muskhelishvili 1953], one will find the following singular integral equation [Chen and Hasebe 1995]:

$$
\frac{1}{\pi} \sum_{k=1}^{N} \int_{L_{k}} \frac{g_{k}^{\prime}(t) d t}{t-t_{o j}}+M\left(t_{o j}\right)+\frac{H}{\pi t_{o j}}=\sigma_{N(p)}\left(t_{o j}\right)+i \sigma_{N T(p)}\left(t_{o j}\right) \quad\left(t_{o j} \in L_{j} ; j=1,2, \ldots, N\right),
$$

where

$$
\begin{aligned}
M\left(t_{o j}\right) & =\frac{1}{2 \pi} \sum_{k=1}^{N} \int_{L_{k}} K_{1}\left(t, t_{o j}\right) g_{k}^{\prime}(t) d t+\frac{1}{2 \pi} \sum_{k=1}^{N} \int_{L_{k}} K_{2}\left(t, t_{o j}\right) \overline{g_{k}^{\prime}(t)} d \bar{t}, \\
K_{1}\left(t, t_{o}\right) & =\frac{d}{d t_{o}}\left\{\ln \frac{t-t_{o}}{\bar{t}-\bar{t}_{o}}\right\}=-\frac{1}{t-t_{o}}+\frac{1}{\bar{t}-\bar{t}_{o}} \frac{d \bar{t}_{o}}{d t_{o}}, \\
K_{2}\left(t, t_{o}\right) & =-\frac{d}{d t_{o}}\left\{\frac{t-t_{o}}{\bar{t}-\bar{t}_{o}}\right\}=\frac{1}{\bar{t}-\bar{t}_{o}}-\frac{t-t_{o}}{\left(\bar{t}-\bar{t}_{o}\right)^{2}} \frac{d \bar{t}_{o}}{d t_{o}}
\end{aligned}
$$

In (23), $\sum_{k=1}^{N}$ ' means that the term corresponding to $k=j$ should be excluded in the summation. This result can be easily seen from the following fact. In (24) and (25), if $t, t_{o}$, and $d t_{o}$ are defined on the $j$-th branch, then $K_{1}\left(t, t_{o}\right)=0$ and $K_{2}\left(t, t_{o}\right)=0$.

In addition, the dislocation distribution $g_{k}^{\prime}(t)$ should satisfy the following single-valued condition of displacements [Chen and Hasebe 1995]:

$$
\sum_{k=1}^{N} \int_{L_{k}} g_{k}^{\prime}(t) d t-H=0
$$

Once the solution for the function $g_{j}^{\prime}(t)$ is obtained from (22) and (26), the SIFs at the branch tip $A_{j}$ can be evaluated by [Savruk 1981; Chen and Hasebe 1995]

$$
\left(K_{1}-i K_{2}\right)_{j}=-\sqrt{2 \pi} \operatorname{Lim}_{t \rightarrow t_{A_{j}}} \sqrt{\left|t-t_{A_{j}}\right|} g_{j}^{\prime}(t) .
$$

On the other hand, from the invariant property for the sum of two normal stresses and (12), at any point we have

$$
\sigma_{T(p)}=4 \operatorname{Re} \phi^{\prime}(z)-\sigma_{N(p)}
$$


In addition, let the point $z$ approach a point $t_{o j}$ on the upper side of $j$-th branch and use the Plemelj formula (see Figure 4b), from (20) and we have

$$
\phi^{\prime+}\left(t_{o j}\right)=\frac{H}{2 \pi t_{o j}}+\frac{i g_{j}^{\prime}\left(t_{o j}\right)}{2}+\frac{1}{2 \pi} \sum_{k=1}^{N} \int_{L_{k}} \frac{g_{k}^{\prime}(t) d t}{t-t_{o j}} \quad\left(t_{o j} \in L_{j} ; j=1,2, \ldots, N\right) .
$$

Under the same condition, or $z \rightarrow t_{o j}$, from (28) and (29), it follows

$$
\sigma_{T(p)}\left(t_{o j}\right)=2 \operatorname{Re}\left(i g_{j}^{\prime}\left(t_{o j}\right)\right)+2 \operatorname{Re}\left(\frac{H}{\pi t_{o j}}+\frac{1}{\pi} \sum_{k=1}^{N} \int_{L_{k}} \frac{g_{k}^{\prime}(t) d t}{t-t_{o j}}\right)-\sigma_{N(p)}\left(t_{o j}\right) .
$$

Substituting (22) into (30) yields

$$
\sigma_{T(p)}\left(t_{o j}\right)=2 \operatorname{Re}\left(i g_{j}^{\prime}\left(t_{o j}\right)\right)+\sigma_{N(p)}\left(t_{o j}\right)-2 \operatorname{Re}\left(M\left(t_{o j}\right)\right)
$$

The T-stress at the branch tip $A_{j}$ in the perturbation field can be defined by (see Figure $4 \mathrm{~b}$ )

$$
T_{j(p)}=\text { Regular part of }\left.\left\{\sigma_{T(p)}\left(t_{o_{j}}\right)\right\}\right|_{t_{o j} \rightarrow t_{A j}} .
$$

It was proved that the term $2 \operatorname{Re}\left(i g_{j}^{\prime}\left(t_{o j}\right)\right)$ is singular when $t_{o j} \rightarrow t_{A j}$ and makes no contribution to the regular part. Therefore, from (31) and (32), the T-stress at the $j$-th branch tip in the perturbation field can be evaluated by $T_{j(p)}=\sigma_{N(p)}\left(t_{A_{j}}\right)-2 \operatorname{Re}\left(M\left(t_{A_{j}}\right)\right)$. Here, $M\left(t_{A j}\right)$ means a value of the integral $M\left(t_{o j}\right)$ defined by (23) when the point $t_{o j}$ is at the branch crack tip point $t_{A_{j}}$.

Considering $\sigma_{N(p)}\left(t_{o_{j}}\right)=-\sigma_{N(u)}\left(t_{o_{j}}\right)$ and using (16) and (17), the T-stress at the branch tip $A_{j}$ in the original field is finally obtained as follows:

$$
T_{j}=\sigma_{T(u)}\left(t_{A_{j}}\right)-\sigma_{N(u)}\left(t_{A_{j}}\right)-2 \operatorname{Re}\left(M\left(t_{A_{j}}\right)\right)
$$

In the numerical solution, the dislocation functions $g_{j}^{\prime}(t)(j=1,2, \ldots, N)$ are generally expressed in the form

$$
g_{j}^{\prime}(t)=\sqrt{\frac{t}{a_{j}-t}} G_{j}(t) \quad\left(0 \leq t<a_{j}\right)
$$

Equation (34) can model the one-half singularity at the crack tip simply because $g_{j}^{\prime}(t)=O\left(a_{j}-t\right)^{-1 / 2}$ as $t \rightarrow a_{j}$. In addition, from (34) we have $g_{j}^{\prime}(t)=O\left(t^{1 / 2}\right)$ as $t \rightarrow 0$. This property may not coincide with the nature of the dislocation distribution at the concave corner. However, it is expected that the influence caused by the assumption $g_{j}^{\prime}(t)=O\left(t^{1 / 2}\right)$ as $t \rightarrow 0$ is minor.

Since the modeling of $-1 / 2$ singularity at the crack tip is the main point in the analysis; it is a generally accepted assumption that the weaker singularity at the kinked corner has no significant influence on the solution of the SIFs and T-stress. Although, this assertion is not easy to prove theoretically. In the literature, many researchers use this assumption in the branch or kinked crack problems [Theocaris 1977; Savruk 1981; Chen and Hasebe 1995]. 
In addition, the following quadrature rules are useful in the numerical solution [Boiko and Karpenko 1981]:

$$
\begin{gathered}
\frac{1}{\pi} \int_{0}^{a} \frac{f(t)}{t-x_{j}} \sqrt{\frac{t}{a-t}} d t=\sum_{m=1}^{M} \frac{w_{m} f\left(t_{m}\right)}{t_{m}-x_{j}} \\
\frac{1}{\pi} \int_{0}^{a} g(t) \sqrt{\frac{t}{a-t}} d t=\sum_{m=1}^{M} w_{m} g\left(t_{m}\right)
\end{gathered}
$$

where

$$
\begin{aligned}
w_{m} & =\frac{a}{M} \sin ^{2}\left(\frac{m \pi}{2 M}\right) \quad(m=1,2, \ldots, M-1), \quad w_{M}=\frac{a}{2 M}, \\
t_{m} & =a \sin ^{2}\left(\frac{m \pi}{2 M}\right) \quad(m=1,2, \ldots, M), \quad x_{j}=a \sin ^{2}\left(\frac{(j-0.5) \pi}{2 M}\right) \quad(j=1,2, \ldots, M) .
\end{aligned}
$$

Here and after, the value $M$ in the quadrature rules (35) and (36) is called the number of integration divisions.

In fact, after the quadrature rule shown by (35) is used for the integral (22), the number of unknowns after discretization of the integral equation is equal to the number of algebraic equations. However, there is one more equation coming from the single-valuedness condition of the displacements. Under this situation, it is suitable to introduce one more unknown, which is shown by the term with $H\left(H=H_{1}+i H_{2}\right)$ in (20) and (21). It is seen that after introducing $H$, the balance of the numbers of unknowns and equations in the relevant algebraic equation is possible [Chen and Hasebe 1995].

Finally, for evaluating the SIFs, one needs to take the following steps: obtain the solution for the dislocation distributions $g_{k}^{\prime}(t)(k=1,2, \ldots, N)$ from the singular integral equations composed of (22) and (26) and obtain the SIFs at branch tips from (27).

In addition, for evaluating T-stress, one needs to take the following steps: obtain the value of $M\left(t_{A_{j}}\right)$ $\left(M\left(t_{A_{j}}\right)=\left.M\left(t_{o j}\right)\right|_{t_{o j}=t_{A_{j}}}\right)$ from (23), evaluate the two stress components $\sigma_{T(u)}\left(t_{A_{j}}\right)$ and $\sigma_{N(u)}\left(t_{A_{j}}\right)$ in the uniform field, and use (33).

From the above analysis, we see that the whole computation depends on the choice of the numbers of integration divisions for the branches. Once the numbers of integration divisions for the branches are assumed, the solution is obtained.

\section{Numerical examination}

In the present case, a kinked crack with kink length $d$ is emanated from the main crack with crack length $2 a$ (see Figure 5). In this case, the two coefficients in (3) need to be revised as follows:

$$
c_{11}(\theta)=\frac{(1+\cos \theta) \cos (\theta / 2)}{2} \sqrt{\frac{a+0.5 d \cos \theta}{a}}, \quad c_{22}(\theta)=\frac{(3 \cos \theta-1) \cos (\theta / 2)}{2} \sqrt{\frac{a+0.5 d \cos \theta}{a}} .
$$

The reason for the modification shown by Equation (39) is as follows. In the case of $\sigma_{x}^{\infty}=\sigma_{y}^{\infty}=p$, we have $K_{1}^{m}=p \sqrt{\pi a}, K_{2}^{m}=0$, and $T^{m}=0$. In addition, if $\theta=0$, from (3) and (39), we have $K_{1}^{k}=p \sqrt{\pi(a+0.5 d)}$. In fact, if the half crack length is $a+0.5 d$, the SIF must take the value $K_{1}^{k}=p \sqrt{\pi(a+0.5 d)}$. Clearly, this result is self-consistent. 


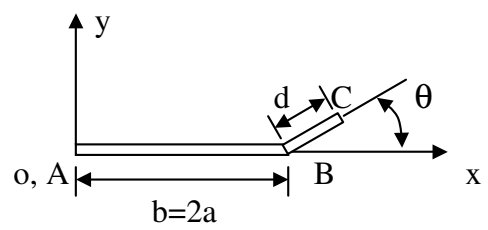

Figure 5. A kinked crack emanating from the main crack.

After substituting $\theta=0$ in (6) and (7), we have $c_{12}(\theta)=0$ and $c_{21}(\theta)=0$. Therefore, the two coefficients $c_{12}(\theta)$, and $c_{12}(\theta)$ will not be affected.

In reality, only small crack kink angles were observed for isotropic materials, for example, a kinked angle of less than 30 degrees. For reference, the computed results up to 60 degrees are presented for the following three numerical examples.

The discretization for (22) and (26) is performed in the following manner. For a kinked crack with $d / b=0.001$ (see Figure 5), we can assume the integration number $M_{1}=5$ (for $d / b=0.001$ ) for the kinked portion, and $M_{2}=135$ for the main crack in (35) and (36). After discretization for (22), we can obtain $2 \times\left(M_{1}+M_{2}\right)$ algebraic equations from (22), which are formulated on $M_{1}+M_{2}$ observing points. Here, the factor of two is coming from the real and imaginary parts of equations. Similarly, after discretization for (26), we can obtain two algebraic equations. Therefore, there are a total of $2 \times\left(M_{1}+M_{2}+1\right)$ equations obtained. In the meantime, there are a total of $2 M_{1}$ unknowns from the kinked portion, or from the $g_{1}^{\prime}(t)$ function at many discrete points. Similarly, there are a total of $2 M_{2}$ unknowns from the main crack portion, or from the $g_{2}^{\prime}(t)$ function at many discrete points. In addition, in (22) and (26), we have assumed two unknowns $H_{1}$ and $H_{2}$ (from $H=H_{1}+i H_{2}$ ). Therefore, the are also a total of $2 \times\left(M_{1}+M_{2}+1\right)$ unknowns.

4.1. Numerical example 1. In the first example, the following conditions are assumed:

$$
\sigma_{x}^{\infty}=p ; \quad \theta=0^{\circ}, 10^{\circ}, \ldots, 60^{\circ} ; \quad d / b=0.001,0.01,0.1,0.25 .
$$

In computation, $M_{1}=5$ for $d / b=0.001, M_{1}=15$ for $d / b=0.01, M_{1}=35$ for $d / b=0.1, M_{1}=55$ for $d / b=0.25$ (the number of integration divisions in (35) and (36) is used for the kinked portion), and $M_{2}=135$ for the main crack portion. The computed results for the SIFs and the T-stress at the kinked crack tip $C$ in Figure 5 are expressed as

$$
\begin{aligned}
K_{1}^{k} & =F_{1}\left(\frac{d}{b}, \theta\right) p \sqrt{\pi(a+0.5 d \cos \theta)}, \\
K_{2}^{k} & =F_{2}\left(\frac{d}{b}, \theta\right) p \sqrt{\pi(a+0.5 d \cos \theta)}, \\
T^{k} & =F_{T}\left(\frac{d}{b}, \theta\right) p .
\end{aligned}
$$

The computed results for the SIFs and the T-stress are plotted in Figures 6-8, respectively.

In addition, for the main crack before kinking we have

$$
K_{1}^{m}=0, \quad K_{2}^{m}=0, \quad T^{m}=p .
$$




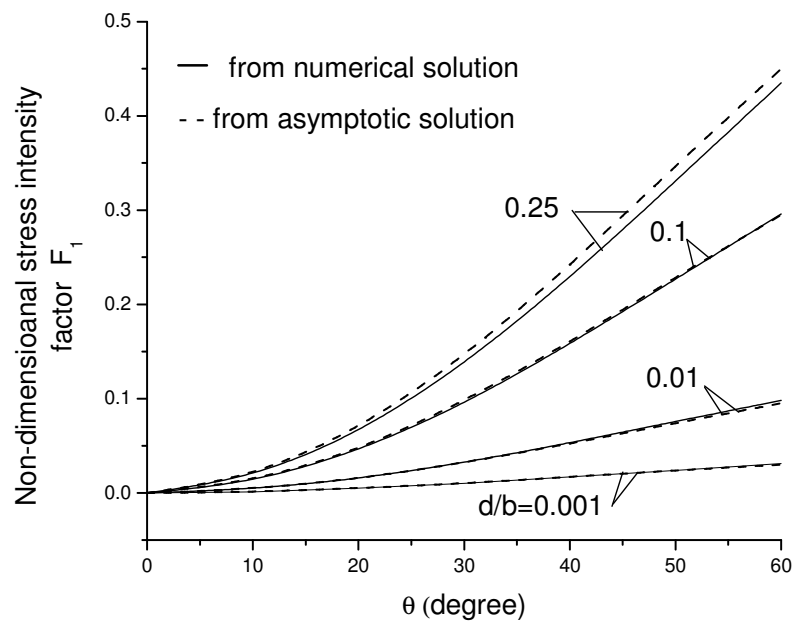

Figure 6. Nondimensional mode I SIF $F_{1}(d / b, \theta)$, from the numerical solution and from the asymptotic solution for a kinked crack under remote loading $\sigma_{x}^{\infty}=p$ (see Figure 5 and Equation (40)).

Further, by using (3)-(5), (7), (8), and (39), the relevant SIFs and the T-stress in the asymptotic solution can also be expressed by the same equations (40)-(42). Those results are also shown in Figures 6-8.

From the plotted results, the following properties have been found. Since $K_{1}^{m}=0$ and $K_{2}^{m}=0$ for the case of the main crack, the values of $K_{1}^{k}$ and $K_{2}^{k}$ are generally small. Secondly, the coincidence for the SIFs from the numerical and asymptotic solutions has been found.

For the values of $K_{1}^{k}$ in the three cases $d / b=0.001,0.01$, and 0.1 , the coincidence is good between the numerical and asymptotic solutions. However, in the case of $d / b=0.25$, some deviations between the two solutions have been found. For the values of $K_{2}^{k}$ in the two cases $d / b=0.001$ and 0.01 , the coincidence is good between the numerical and asymptotic solutions. However, in the cases $d / b=0.1$ and 0.25 , some deviations between the two solutions have been found, particularly for $\theta \geq 50^{\circ}$. For the values of $T^{k}$ in the four cases $d / b=0.001,0.01,0.1$, and 0.25 , the computed results from the numerical solutions merge into one curve, and the results deviate slightly from those from the asymptotic solution.

4.2. Numerical example 2. In the second example, the remote loading is $\sigma_{y}^{\infty}=p$ and the other computation conditions are same as in the first example. The computed results for the SIFs and the T-stress at the kinked crack tip $C$ in Figure 5 are expressed by the same equations (40)-(42). The computed results for the SIFs and the T-stress are plotted in Figures 9-11, respectively.

In addition, for the main crack before kinking we have

$$
K_{1}^{m}=p \sqrt{\pi a}, \quad K_{2}^{m}=0, \quad T^{m}=-p .
$$

Further, by using (3)-(5), (7), (8), and (39), the relevant SIFs and the T-stress in the asymptotic solution can also be expressed by the same equations (40)-(42). Those results are also shown in Figures 9-11.

For the values of $K_{1}^{k}$ in the two cases $d / b=0.001$, and 0.01 , the coincidence is good between the numerical and asymptotic solutions. However, in the cases of $d / b=0.1$ and 0.25 , some deviations between the two solutions have been found. For the values of $K_{2}^{k}$ in the four cases $d / b=0.001,0.01$, 


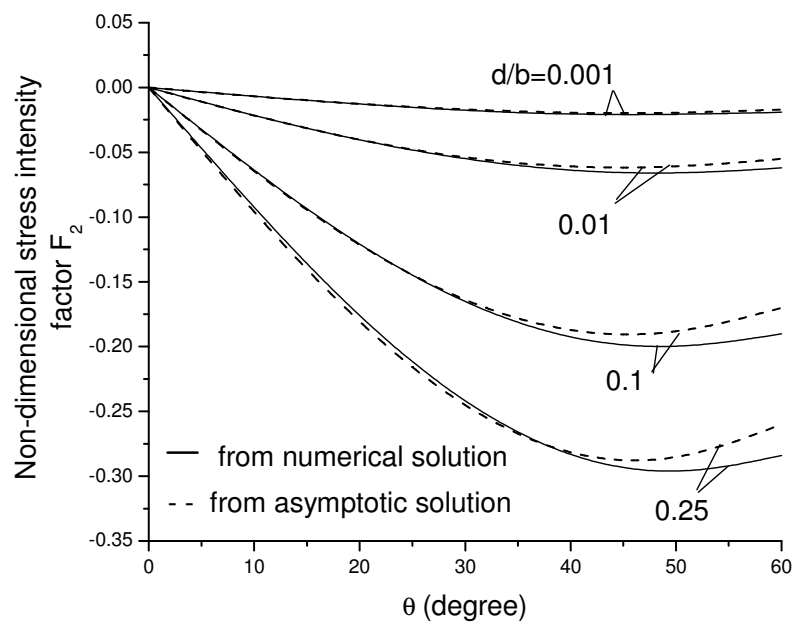

Figure 7. Nondimensional mode II SIF $F_{2}(d / b, \theta)$, from the numerical solution and from the asymptotic solution for a kinked crack under remote loading $\sigma_{x}^{\infty}=p$ (see Figure 5 and Equation (41)).

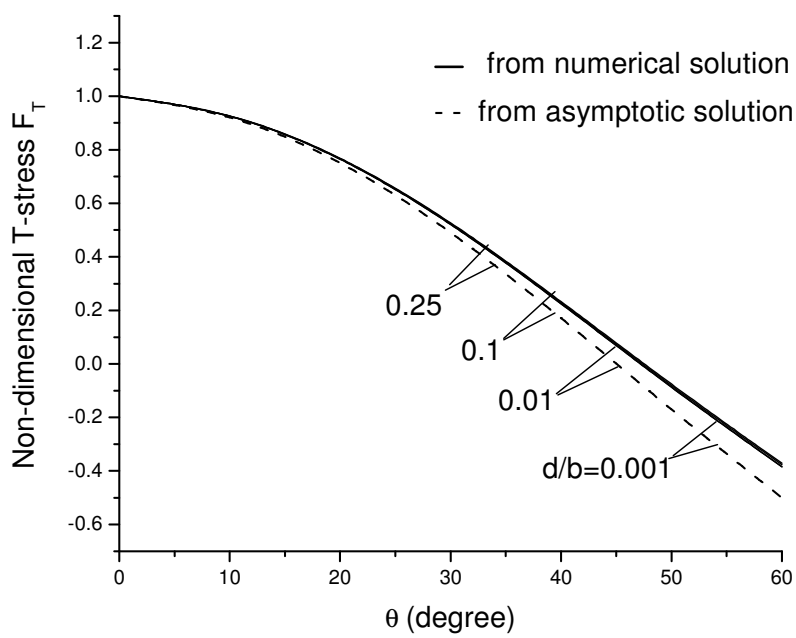

Figure 8. Nondimensional T-stress $F_{T}(d / b, \theta)$, from the numerical solution and from the asymptotic solution for a kinked crack under remote loading $\sigma_{x}^{\infty}=p$ (see Figure 5 and Equation (42)).

0.1 , and 0.25 , the coincidence is good between the numerical and asymptotic solutions. For the values of $T^{k}$ in the two cases $d / b=0.001$ and 0.01 , the coincidence is good between the numerical and asymptotic solutions. However, in the cases of $d / b=0.1$ and 0.25 , some deviations between the two solutions have been found.

4.3. Numerical example 3. In the third example, the remote loading is $\sigma_{x y}^{\infty}=-p$ and the other computation conditions are same as in the first example. The computed results for the SIFs and the T-stress at 
the kinked crack tip $C$ in Figure 5 are expressed by the same equations (40)-(42). The computed results for the SIFs and the T-stress are plotted in Figures 12-14, respectively.

In addition, for the main crack before kinking we have

$$
K_{1}^{m}=0, \quad K_{2}^{m}=-p \sqrt{\pi a}, \quad T^{m}=0 .
$$

Further, by using Equations (3)-(5), (7), (8), and (39), the relevant SIFs and the T-stress in the asymptotic solution can also be expressed by the same Equations (40)-(42). Those results are also shown in Figures $12-14$.

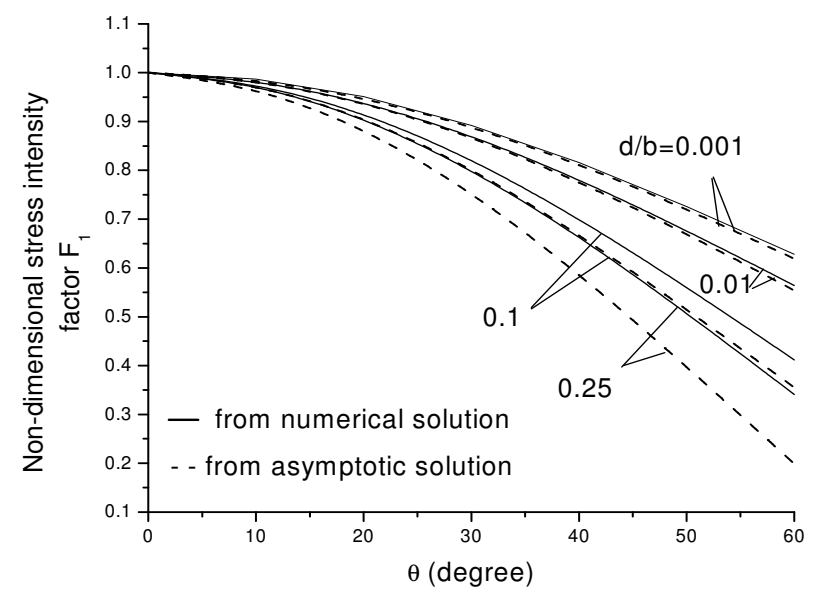

Figure 9. Nondimensional mode I SIF $F_{1}(d / b, \theta)$, from the numerical solution and from the asymptotic solution for a kinked crack under remote loading $\sigma_{y}^{\infty}=p$ (see Figure 5 and Equation (40)).

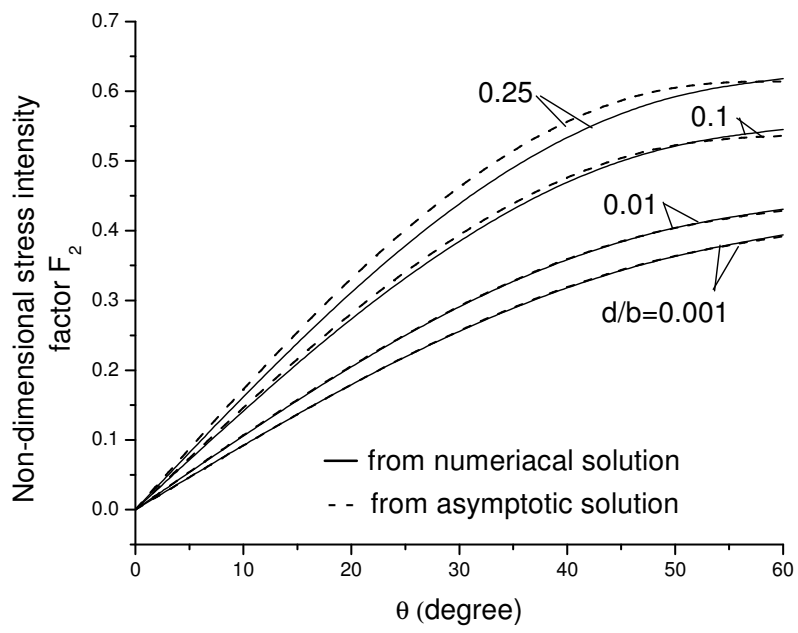

Figure 10. Nondimensional mode II SIF $F_{2}(d / b, \theta)$, from the numerical solution and from the asymptotic solution for a kinked crack under remote loading $\sigma_{y}^{\infty}=p$ (see Figure 5 and Equation (41)). 


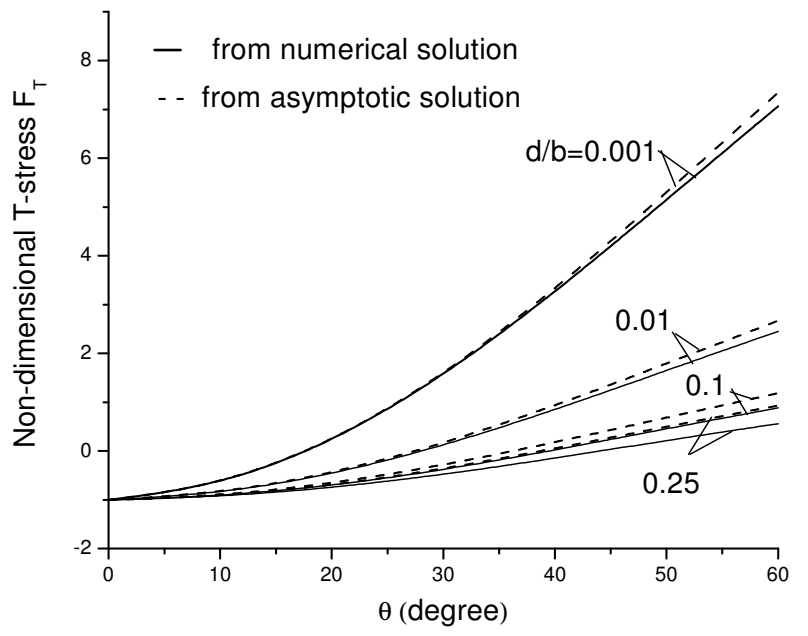

Figure 11. Nondimensional T-stress $F_{T}(d / b, \theta)$, from the numerical solution and from the asymptotic solution for a kinked crack under remote loading $\sigma_{y}^{\infty}=p$ (see Figure 5 and Equation (42)).

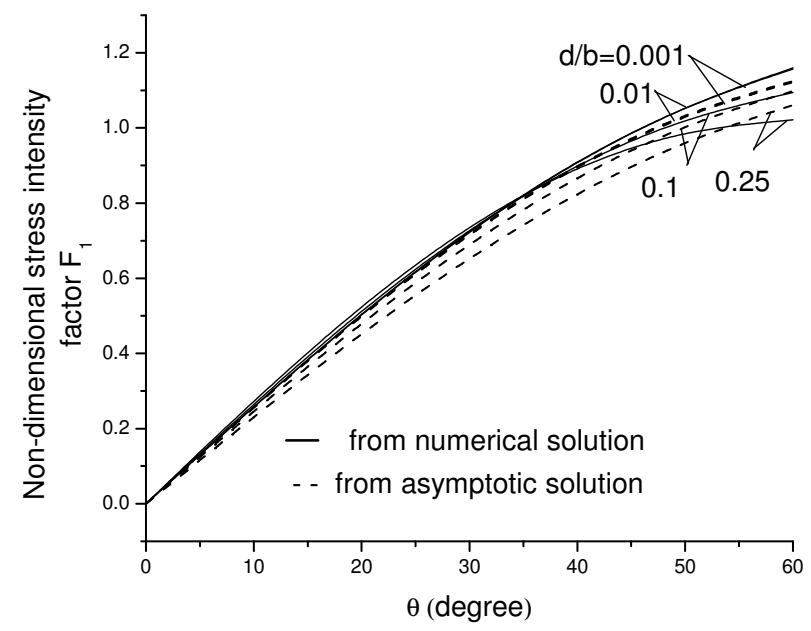

Figure 12. Nondimensional mode I SIF $F_{1}(d / b, \theta)$, from the numerical solution and from the asymptotic solution for a kinked crack under remote loading $\sigma_{x y}^{\infty}=-p$ (see Figure 5 and Equation (40)).

For the values of $K_{1}^{k}$ in the four cases $d / b=0.001,0.01,0.1$, and 0.25 , the coincidence is good between the numerical and asymptotic solutions. For the values of $K_{2}^{k}$ in the four cases $d / b=0.001$, $0.01,0.1$, and 0.25 , some deviations between the two solutions have been found. Particularly, the values of $K_{2}^{k}$ in the asymptotic solution do not depend on the ratio $d / b$, and it is not reasonable. For the values of $T^{k}$ in the two cases $d / b=0.001$ and 0.01 , some deviations between the two solutions have been found. However, in the cases of $d / b=0.1$ and 0.25 , the coincidence is good between the numerical and asymptotic solutions. 


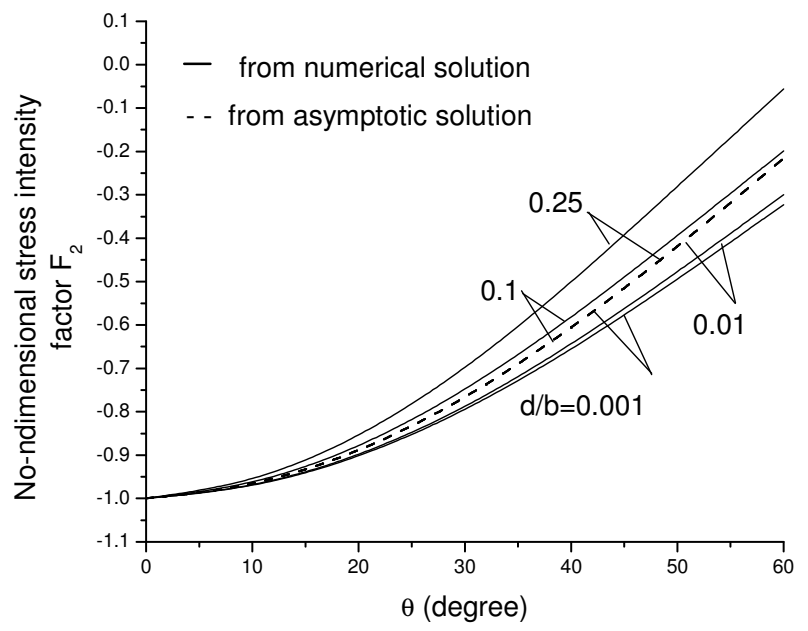

Figure 13. Nondimensional mode II SIF $F_{2}(d / b, \theta)$, from the numerical solution and from the asymptotic solution for a kinked crack under remote loading $\sigma_{x y}^{\infty}=-p$ (see Figure 5 and Equation (41)).

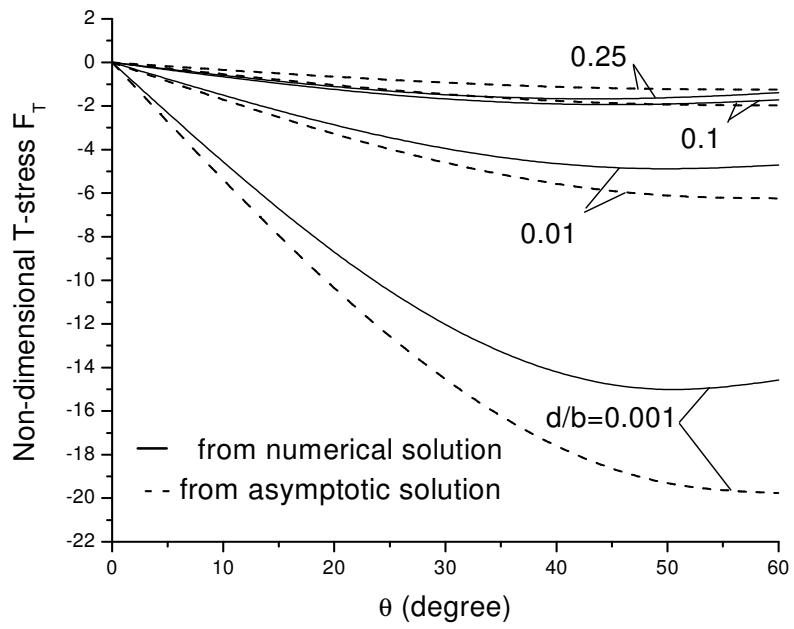

Figure 14. Nondimensional T-stress $F_{T}(d / b, \theta)$, from the numerical solution and from the asymptotic solution for a kinked crack under remote loading $\sigma_{x y}^{\infty}=-p$ (see Figure 5 and Equation (42)).

\section{Conclusion}

In this paper, an efficient numerical solution for a kinked crack with a finite kink has been developed. The aim of the present study is to examine the accuracy in the solutions for the stress intensity factors (SIFs) and T-stress from the asymptotic solution. A particular advantage of the asymptotic solution is that it provides an explicit form for the solutions. However, the asymptotic solution itself cannot examine the accuracy achieved in the solution. The suggested numerical solution will give a full examination of the results obtained in the asymptotic solution. 
In this paper, the computed results for the SIFs and T-stress for the studied problem are given. From the computed results in the numerical solution and the results in the asymptotic solution, the following conclusion can be reached. For a configuration of the kinked crack within the ranges: $d / b=0.001$, $0.01,0.1$ and 0.25 and $0^{\circ} \leq \theta \leq 60^{\circ}$ (see Figure 5); and for the remote loadings: $\sigma_{x}^{\infty}=p, \sigma_{y}^{\infty}=p$, or $\sigma_{x y}^{\infty}=-p$, the asymptotic solution can provide a qualitatively correct solution. This situation can be seen from Figures 6-14.

However, under some conditions, the asymptotic solution cannot provide quantitatively accurate results. For the SIFs, it is expected that the asymptotic solution can provide a reasonable results only for the following ranges: for a configuration of the kinked crack within the range: $d / b<0.01$ and $0^{\circ} \leq \theta \leq 20^{\circ}$ (see Figure 5); and for the remote loadings: $\sigma_{x}^{\infty}=p, \sigma_{y}^{\infty}=p$, or $\sigma_{x y}^{\infty}=-p$. For the computed values of the T-stresses, the situation is not the same as in the case of the SIFs. For example, in the conditions $d / b<0.01, \theta \geq 30^{\circ}$, and $\sigma_{x y}^{\infty}=-p$, the asymptotic solutions for the T-stress shown in Figure 14 cannot provide accurate results.

\section{References}

[Betegón and Hancock 1991] C. Betegón and J. W. Hancock, "Two-parameter characterization of elastic-plastic crack-tip fields", J. Appl. Mech. (ASME) 58:1 (1991), 104-110.

[Boiko and Karpenko 1981] A. V. Boiko and L. N. Karpenko, "On some numerical methods for the solution of the plane elasticity problem for bodies with cracks by means of singular integral equation”, Int. J. Fract. 17:4 (1981), 381-388.

[Broberg 2005] K. B. Broberg, "A note on T-stress determination using dislocation arrays", Int. J. Fract. 131:1 (2005), 1-14.

[Chen 2004] Y. Z. Chen, "Solution of integral equation in curve crack problem by using curve length coordinate", Eng. Anal. Bound. Elem. 28:8 (2004), 989-994.

[Chen and Hasebe 1995] Y. Z. Chen and N. Hasebe, "New integration scheme for the branch crack problem", Eng. Fract. Mech. 52:5 (1995), 791-801.

[Cotterell and Rice 1980] B. Cotterell and J. R. Rice, "Slightly curved or kinked cracks", Int. J. Fract. 16:2 (1980), 155-169.

[Dutta et al. 1991] B. K. Dutta, A. Kakodkar, and S. K. Maiti, "Two singular points finite elements in the analysis of kinked cracks", Comput. Mech. 7:5-6 (1991), 329-339.

[Fett 2001] T. Fett, "Stress intensity factors and $T$-stress for internally cracked circular disks under various boundary conditions", Eng. Fract. Mech. 68:9 (2001), 1119-1136.

[Fett et al. 2006] T. Fett, G. Rizzi, and H.-A. Bahr, "Green's functions for the $T$-stress of small kink and fork cracks”, Eng. Fract. Mech. 73:10 (2006), 1426-1435.

[Karihaloo and Xiao 2001] B. L. Karihaloo and Q. Z. Xiao, "Higher order terms of the crack tip asymptotic field for a notched three-point bend beam", Int. J. Fract. 112:2 (2001), 111-128.

[Larsson and Carlsson 1973] S. G. Larsson and A. J. Carlsson, "Influence of non-singular stress terms and specimen geometry on small-scale yielding at crack tips in elastic-plastic materials", J. Mech. Phys. Solids 21:4 (1973), 263-277.

[Li and Xu 2007] X.-F. Li and L. R. Xu, "T-stresses across static crack kinking”, J. Appl. Mech. (ASME) 74:2 (2007), 181-190.

[Lo 1978] K. K. Lo, “Analysis of branched cracks”, J. Appl. Mech. (ASME) 45:4 (1978), 797-802.

[Melin 1986] S. Melin, “On singular integral equations for kinked cracks”, Int. J. Fract. 30:1 (1986), 57-65.

[Melin 1994] S. Melin, "Accurate data for stress intensity factors at infinitesimal kinks”, J. Appl. Mech. (ASME) 61:2 (1994), 467-470.

[Melin 2002] S. Melin, "The influence of the T-stress on the directional stability of cracks", Int. J. Fract. 114:3 (2002), 259265.

[Muskhelishvili 1953] N. I. Muskhelishvili, Some basic problems of the mathematical theory of elasticity, Noordhoff, Groningen, 1953. 
[Rice 1974] J. R. Rice, "Limitations to the small scale yielding approximation for crack tip plasticity", J. Mech. Phys. Solids 22:1 (1974), 17-26.

[Savruk 1981] M. P. Savruk, Two-dimensional problems of elasticity for body with crack, Naukova Dumka, Kiev, 1981. In Russian.

[Theocaris 1977] P. S. Theocaris, “Asymmetric branching of cracks”, J. Appl. Mech. (ASME) 44:4 (1977), 611-618.

[Williams 1957] M. L. Williams, "On the stress distribution at the base of a stationary crack", J. Appl. Mech. (ASME) 24:1 (1957), 109-114.

[Xiao and Karihaloo 2002] Q. Z. Xiao and B. L. Karihaloo, "Approximate Green's functions for singular and higher order terms of an edge crack in a finite plate", Eng. Fract. Mech. 69:8 (2002), 959-981.

[Yang and Ravi-Chandar 1999] B. Yang and K. Ravi-Chandar, "Evaluation of elastic T-stress by the stress difference method", Eng. Fract. Mech. 64:5 (1999), 589-605.

Received 24 Sep 2008. Revised 24 Jun 2009. Accepted 4 Jul 2009.

Y. Z. CHEN: chens@ujs.edu.cn

Division of Engineering Mechanics, Jiangsu University, Xue Fu Road 301, Zhenjiang, Jiangsu 212013, China

X. Y. LIN: xiaoyun39527@yahoo.com

Division of Engineering Mechanics, Jiangsu University, Xue Fu Road 301, Zhenjiang, Jiangsu 212013, China

Z. X. WANG: wzx-5566@163.com

Division of Engineering Mechanics, Jiangsu University, Xue Fu Road 301, Zhenjiang, Jiangsu 212013, China 